\title{
Malignant Pleural Mesothelioma with No Asbestos Exposure-A Case Report
}

\author{
Authors \\ Dr Bhabani Sankar Rout, Prof. Dr Pravati Dutta, Asso. Prof. Dr Rekha Manjhi, \\ Asso. Prof. Dr Sudarsan Pothal, Asst. Prof. Dr Aurobindo Behera, \\ Asst. Prof. Dr Gourahari Pradhan \\ Department of Pulmonary Medicine, VSSIMSAR, Burla
}

\begin{abstract}
Malignant Mesothelioma is an aggressive primary malignant tumor of the pleura, usually caused by prolong asbestos exposure. The prognosis of malignant pleural mesothelioma is very poor and median survival ranges from 8-14 months from diagnosis. There is a long latency period of development of the cancer from asbestos exposure. The absence of an explicit asbestos exposure is another diagnostic problem.
\end{abstract}

Case Report: A 60 year old male average body built with BMI $18.6 \mathrm{Kg} / \mathrm{m} 2$, farmer by occupation, non smoker, ex alcoholic presented with chief complain of right sided chest pain for 2 month, SOB for 1 month, dry cough for Imonth, with past h/o trauma by iron rod to right side of chest. No h/o asbestos exposure. No significant past history and family history. Examination-Vitals are normal. Pallor 1+, grade 2 Clubbing, no lymphadenopathy. Respiratory examination reveals decrease chest movement, stony dull on percussion, grossly diminished breath sound on right hemi thorax with slightly deviated mediastinum to contralateral side. Investigation -Complete blood haemogram normal except leucocytosis. CXR-Right massive pleural effusion with contralateral mediastinal shift, USG-right massive pleural effusion with pleural thickening with normal abdomen pelvis. CECT THORAX-Nodular thickening of mediastinal and right parietal pleura which takes enhancement with right gross pleural effusion. Pleural fluid reveals cytology show lymphocytosis and no malignant cell found. Biochemical test found low ADA exudative with high LDH, THORACOSCOPY -Gross appearance showed multiple white and grey nodules over both visceral and parietal pleura. Biopsy taken and $h / p$ study confirms malignant mesothelioma. Patients referred to radiotherapy department for further management.

Conclusion: Though patient has no history of exposure to asbestos. So other risk factor present in the soil or his firm may be responsible for the development of malignant Mesothelioma.

Keywords: Mesothelioma, Thoracocentesis, Thoracoscopy and Mediastinum.

\section{Background}

Mesothelioma is a rare neoplasm of mesothelial cell of pleura, found in approx. 1 case per million per year. It arises from mesothelial cells lining the visceral cavity eg pleura, peritoneum, pericardium and tunica vaginallis. Malignant pleural mesothelioma is most common among them. It develops mainly in individual of $5^{\text {th }}$ to $7^{\text {th }}$ decade. It affects mainly male gender. Its main risk factor is asbestos exposure. The incidence of mesothelioma in people with asbestos exposure is 300 times higher than the general population ${ }^{5}$. Other risk factors include erionite, radiotherapy, surgery causing pleural scarring and simian virus 
40. There is a long latency period of development of malignancy from exposure. The most common symptoms are chest pain, dyspnoea, cough and breathlessness. Diagnostic imaging involves: standard chest $\mathrm{X}$ rays, CT, MRI, and PET scan of thorax. The best method for final diagnosis is video assisted thoracoscopy followed by pleural biopsy and histopathology study. Treatment of mesothelioma includes chemotherapy, radiotherapy, immunotherapy and surgical resection. The mortality is almost $100 \%$, Recovery has been rarely reported. The median survival duration is 8 to 12 months ${ }^{4}$.

\section{Case Report}

A 60 yr old male with average body built and BMI $-18.6 \mathrm{Kg} / \mathrm{m} 2$ presented to the department of pulmonary medicine of our hospital with complaint of chest pain for 2 month, SOB for 1 month, dry cough for 1 month. Personal history: He is an exalcoholic, non-smoker, farmer in occupation, living in rural area, with no hlo asbestos exposure. Past history of trauma to chest (Rt) with penetration of iron rod due to fell down from a tree for which ICT was given and he has been treated successfully. No other significant past history and family history. On physical examination, It is found that vitals are normal, pallor 1+, Grade 2 clubbing, no lymphadenopathy. Respiratory system examination reveals decrease chest movement, decrease vocal fremitus, stony dull on percussion, with grossly diminished breath sound on right hemi thorax with slightly deviated mediastinum to contra-lateral side.

\section{Investigation}

Complete blood haemogram is normal except leucocytosis. Sputum for AFB- Negative, sputum for CBNAAT- M. Tuberculosis not detected, CXR finding shows right massive pleural effusion with contra lateral mediastinal shift. Ultrasonography of thorax and abdomen shows right gross pleural effusion with pleural thickening and normal abdomen and pelvis. CECT thorax shows nodular thickening of mediastinal and right costal parietal pleura and taking enhancement with right pleural effusion.

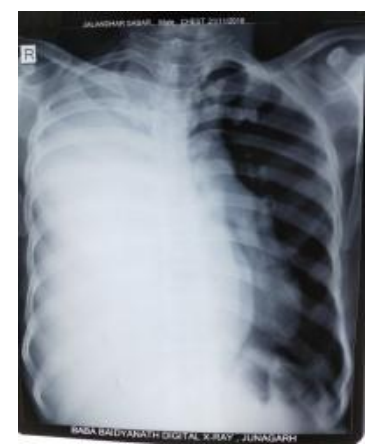

Figure 1- PA view of chest showing massive pleural effusion with contralateral mediastinal shift.

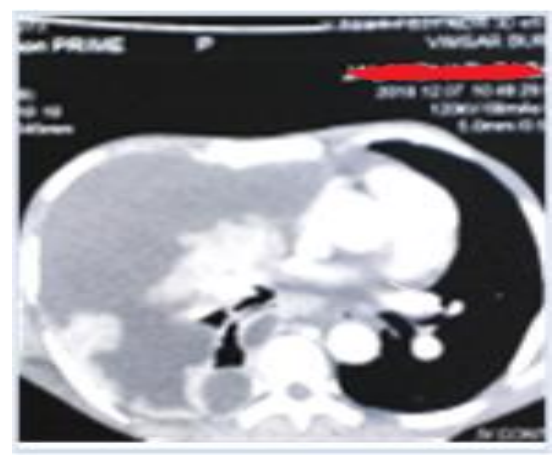

Figure 2 CT scan thorax shows right PLEF with nodular and parietal pleural thickening and mediastinal shifting

Pleural fluid- Hemorrhagic in color, cytology shows lymphocytosis without any malignant cell, pleural fluid for AFB-negative. Biochemical test reveals ADA-38 U/L, protein-4.8 gm/dl, LDH $1385 \mathrm{U} / \mathrm{L}$ and Pleural fluid CBNAAT is $\mathrm{M}$. tuberculosis not detected. There are few serum markers which help in diagnosis and prognosis of the disease.

Thoracoscopy showed multiple grays and white nodules over both visceral and parietal pleura on gross morphology. And Thoracoscopy guided biopsy taken and on hlp study showedfibrocollagenous stroma covered by wide uneven band round and elongated neoplastic cell suggestive of malignant mesothelioma of mixed histological pattern. 


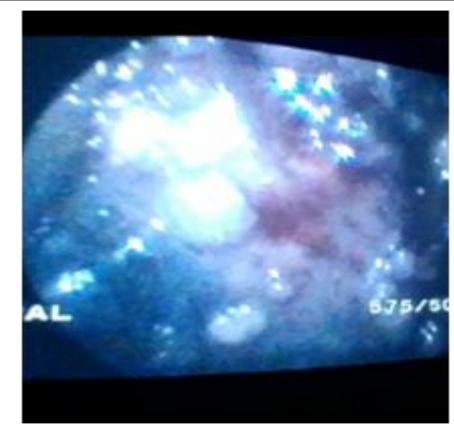

Figure 3 Pleural nodule on thoracoscopy

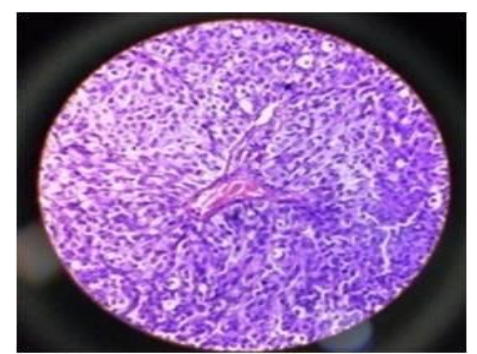

Figure 4 h/p study showing fibrocartilegnous strand with malignant cell.

\section{Intervention}

This patient presented with Rt massive pleural effusion and repeated thoracocentesis done appx 6lit. Thoracoscopy was done for repeated accumulation of fluid and biopsy taken followed by pleurodesis is done with TALC insufflations and ICT insertion.

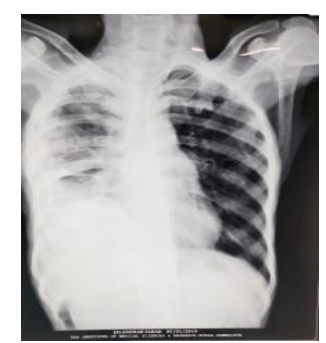

Figure 5 PA view of thorax after talc pleurodesis

\section{Discussion}

Malignant mesothelioma is a primary neoplasm of the mesothelial tissues present in the pleura, peritoneum, pericardium, and tunica vaginalis of testis. It is most commonly seen in individual who have been exposed to asbestos directly or indirectly. The incidence of malignant mesothelioma is increasing and usually diagnosed at the late advance stage because of the long latency period (30years) of asbestos exposure. The factors that are contributed to the delay in establishing Mesothelioma includes the limited workplace epidemiologic data and the misclassification of most reported cases as other tumors $^{8}$. Diffuse malignant mesothelioma needs to be distinguished from the less-common focal benign mesothelioma and metastatic adenocarcinoma. These pleural tumors, which are not related to asbestos exposure, have good prognosis and often do not recur after surgical resection. Malignant pleural Mesothelioma is a rare tumor of prevalence 7- 13 per million per year $^{2}$ and still rarer in India. Only 15 case reported in last 25 year $^{3}$ in population unexposed to asbestos is still rarer, with 1-2 per million per year $^{3}$ in a year with male to female ratio $2.6: 1^{4}$, The postulated correlation in non exposed asbestos case is operation of other carcinogen, genetic factor and viral infection (SV-40 virus). Histologically ${ }^{4}$ Malignant pleural mesothelioma is 4 types 1.epithelial, 2.sarcomatoid, 3.mixed variety and 4. Desmoids variety. The rarity of occurrence and the absence of exposure to asbestos and unusual presentation prompted us to report the case. The prognosis of malignant mesothelioma is very poor and the median survival period is $8-14$ month from diagnosis. ${ }^{4}$

\section{Treatment and Prognosis}

The prospect for curative treatment is not good. The treatments available are surgery, radiotherapy, and chemotherapy. EPP (extra pleural pnemonectomy) is a radical surgical procedure involving complete removal of the ipsilateral lung along with parietal and visceral pleura, pericardium with portion of phrenic nerve and the majority of the hemidiaphragm. MPM is a radio resistant neoplasm. Radiation therapy may play a role in preventing chest wall recurrence after invasive procedure, and improving local control after pleurectomy or extra pleural pnemonectomy. Other therapy like immunotherapy, targeted therapy and gene therapy are in trial for the management of malignant mesothelioma. Palliative treatment to control the symptom with analgesia and preventing recurrent pleural effusion by 
pleurodesis with TALC insufflations. Most patients with pleural mesothelioma, whether treated or untreated, will die of complications of local disease like Increasing bulk of the tumor, deterioration of lung function, and compromise the respiratory function. Respiratory failure is the major cause of mortality despite the fact that as many as $82 \%$ of patients have distant metastases at the time of autopsy ${ }^{6}$. Frequent sites of metastases are the liver, adrenal gland, kidney, and contra lateral lung. Intracranial metastases have been reported but are rare. The International Union against Cancer proposed a tumor node metastasis (TNM) staging system that evolved into the presently described international mesothelioma Interest Group (IMIG) staging system and Butchart staging system. The IMIG staging system is the only system that has been validated in two large surgical series of Mesothelioma. Operative intervention in Mesothelioma is categorized as ${ }^{6}$ : (1) primary effusion control, (2) Cytoreduction before multimodal therapy, or (3) delivery and monitoring of innovative modes of intrapleural therapy. This patient was transferred to radiotherapy department for further management.

\section{Conclusion}

Though this patient has no history of exposure to asbestos, So other risk factor, like any carcinogen present in the soil or his firm, (erionite) genetic factor, or hlo trauma by iron rod may be responsible for the development of malignant mesothelioma. Early intervention with medical Thoracoscopy and biopsy will help in early diagnosis and management. A significant increase in survival among MPM patients can be reached through early diagnosis of the disease. After confirmation of diagnosis as malignant pleural Mesothelioma with stage IV, the patient was transferred to radiotherapy department for further management.

\section{Reference}

1. Rao. Sukhesh Malignant Mesothelioma, A Case Report, Lung India Journal, 200; 26;53-54.

2. Patel SN Kether Malignant Pleural Mesothelioma, A Case Report J Manipulative ,2005;28;726

3. Light Richard $\mathrm{W}$, Pleural Disease, $6^{\text {th }}$ ed,2014; 11;189-199

4. Grippi Michael A et al fishman pulmonary disease and disorder, $5^{\text {th }}$ edition, ch 10 1216-27.

5. Seaton Anthony, Seaton Douglas and Leitch A Gordon, Crofton and douglas respiratory disease, $5^{\text {th }}$ edition, -1176 .

6. J Barrerio Timothy, Katzman Philip j, JAOA CASE REPORT, malignant mesothelioma: a case presentation vol106.no 12.dec 2006. 